\title{
Stability of the equilibrium positions of an engine with nonlinear quadratic springs
}

\author{
Nicolae-Doru Stănescu ${ }^{1 *}$, Dinel Popa ${ }^{1 \dagger}$ \\ 1 University of Pitești, 1, Târgul din Vale, 110040, Romania
}

Received 15 July 2013; accepted 04 November 2013

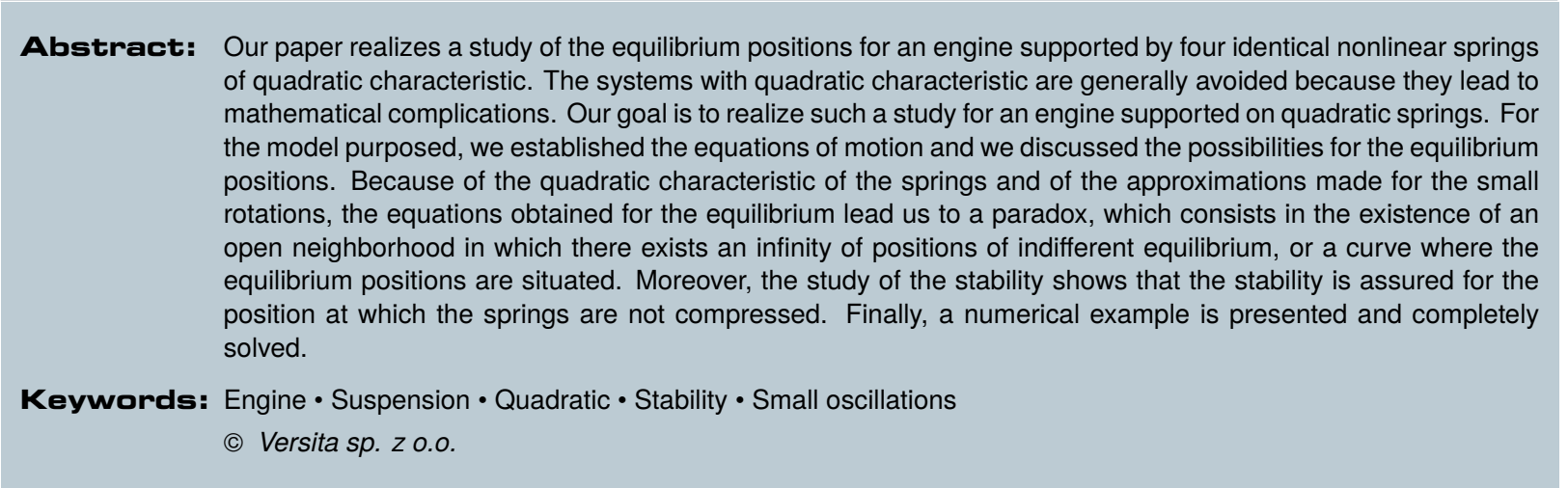

\section{Introduction}

The vibrations of an engine are usually studied using models with linear elastic springs. These springs are always pre-tensioned by a sufficient large force so that the contact between the engine and springs is not lost [1-6]. This model is used due to its simplicity obtained by the linear relation between the force and the displacement given by the elastic springs. The study is also performed using CAE soft [7]. Other authors [8] identify causes of the engine vibrations and studied the relations between different parameters. The authors of this paper studied

*E-mail: s_doru@yahoo.com

†E-mail: dinel_popa@yahoo.com the vibrations of the engine supported by neo-Hookean springs [9]. The study on nonlinear models [9] are performed using special characteristics of the springs. The use of quadratic characteristic is avoided because of the complications that appear (even if the second degree equation is easily solved) and the fact that the characteristic is not one with symmetries relative to origin. This absent of symmetries makes the written of the equations of motion a very complicate and laborious process.

Kilic et al. [10] discuss the problem of two quadratic springs antagonistically coupled obtaining a spring with linear mechanical adjustable characteristic. Luo et al. [11] study the leaf springs using a multibody approach and apply the theory to Hotchkiss rigid axle type, tires and a leaf-spring model. Champion and Champion [12] obtain a wave spring type equation for a small amplitude, 
undamped extensional oscillation of a spring mass system, where the spring is characterized by a quadratic force extension relation. Excellent overview for the synthesis of nonlinear springs and their design can be found in $[13,14]$. Worden et al. [15] perform an overview of a number of nonlinear identification methods, specially to nonlinear automotive dampers. Poussot-Vassal et al. [16] perform an overview of the semi-active suspension control strategy performances. Yang et al. [17] treat the problem of the robust control of certain systems using DOBC (disturbance observer based control) with applications to MAGLEV suspension. Lee and Han [18] use CAE to automotive structure development. Huang et al. [19] discuss an algorithm for an active suspension for a half-car. Huang and Chen [20] use the fuzzy theory for the control of the automotive suspension. Koch et al. [21] study the control of the active suspension with continuously variable damper. Ibrahim [22] performs a review in nonlinear passive vibration isolators discussing the chaos, the influence of the random vibrations, vibration protection under vibro-impact etc. Wei and Yuling [23] study the nonlinear vibrations of the automotive with magneto-rheological damper. Surace et al. [24] present an shock absorber with nonloinear damping. Ando and Suzuki [25] discuss an active suspension system with nonlinear characteristic. Lin and Kanellakopoulos [26] present a nonlinear back-stepping design for the control of active suspension systems.

Our paper is organized as follows: first of all we described the model used in the paper, then we obtain the equations of motion. In the next paragraph we study the equilibrium positions and then the stability of these positions. A numerical example is also presented. The paper ends with conclusions.

\section{The model}

The model considered in this paper is drawn in Figure 1. It consists in the shell $A B D E$ which schematized the engine block. The shell is considered homogeneous of dimensions $2 l_{1}$ and $2 l_{2}$, respectively, and it is linked to the ground by four identical springs. We denote by $C$ the center of the shell, which is also the center of gravity. The axes $C x, C y$ and $C z$ are the principal central axes of inertia. the system is assumed to have three degrees of freedom: the vertical displacement $z$ of the center of gravity $C$, the rotation of angle $\psi$ about the $C x$-axis, and the rotation of angle $\theta$ about the $C y$-axis. Both rotations are assumed to be small, so that the following approximations take place: $\sin \psi \simeq \psi, \cos \psi \simeq 1, \sin \theta \simeq$ $\theta, \cos \theta \simeq 1$. The mechanical parameters $m$ - the mass of

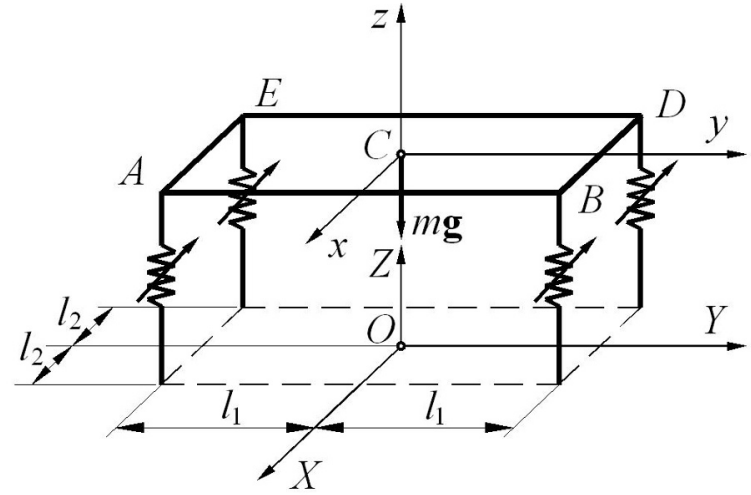

Figure 1. Mathematical model.

the shell, and the principal central moments of inertia $J_{x}$, and $J_{y}$, respectively, with respect to the axes $C x$, and $C y$, respectively are known.

The elastic forces in the springs are given by the nonlinear expression

$$
\digamma=k_{1} \delta+k_{2} \delta^{2}
$$

where $\delta$ is the elongation of the spring.

With these assumptions the elastic forces which act at the points $A, B, D$ and $E$ are

$$
\begin{aligned}
& F_{A}=k_{1}\left(z-l_{1} \psi-l_{2} \theta\right)+k_{2}\left(z-l_{1} \psi-l_{2} \theta\right)^{2}, \\
& F_{B}=k_{1}\left(z+l_{1} \psi-l_{2} \theta\right)+k_{2}\left(z+l_{1} \psi-l_{2} \theta\right)^{2}, \\
& F_{D}=k_{1}\left(z+l_{1} \psi+l_{2} \theta\right)+k_{2}\left(z+l_{1} \psi+l_{2} \theta\right)^{2}, \\
& F_{E}=k_{1}\left(z-l_{1} \psi+l_{2} \theta\right)+k_{2}\left(z-l_{1} \psi+l_{2} \theta\right)^{2} .
\end{aligned}
$$

\section{Equations of motion}

The theorem of momentum leads to the equation

$$
m \ddot{z}=F_{A}+F_{B}+F_{D}+F_{E}-m g,
$$

where $g$ is the gravitational acceleration, and the forces are taken with their signs corresponding to their projections onto the $C z$-axis.

The theorem of moment of momentum offers the following two equations, relative to the $C x$ and $C y$ axes:

$$
\begin{aligned}
& J_{x} \ddot{\psi}=-F_{A} l_{1}+F_{B} l_{1}+F_{D} l_{1}-F_{E} l_{1}, \\
& J_{y} \ddot{\theta}=-F_{A} l_{2}-F_{B} l_{2}+F_{D} l_{2}+F_{E} l_{2} .
\end{aligned}
$$




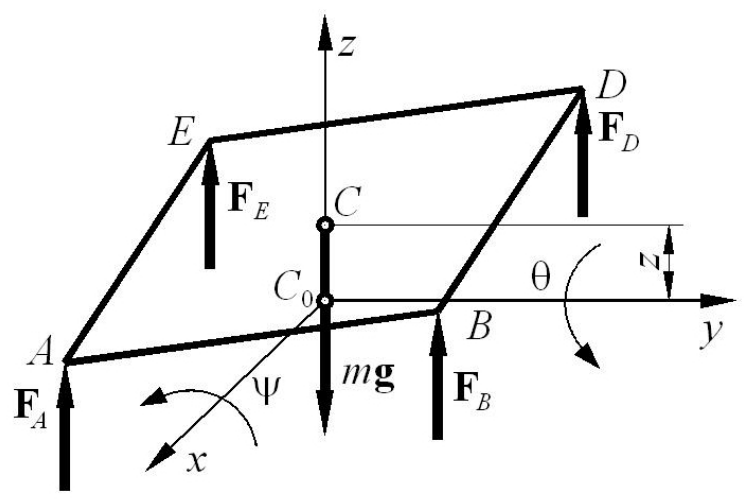

Figure 2. The equilibrium positions.

The equations (3) and (4) hold true if and only if the forces $F_{A}, F_{B}, F_{C}$ and $F_{D}$ are vertical ascendent. If the sense of one or more of these forces is vertical descendent the equations must be modified correspondingly. The problem appears because the expressions of the elastic forces in the springs are quadratic and they are not odd, that is the origin is not a point of symmetry.

\section{The equilibrium positions}

\subsection{All the elastic forces are vertical ascendent}

Considering that the elastic forces are vertical ascendent (Figure 2), the equations from which one determines the equilibrium positions read

$$
\begin{aligned}
& F_{A}+F_{B}+F_{D}+F_{E}=m g, \\
& F_{A} l_{1}+F_{E} l_{1}=F_{B} l_{1}+F_{D} l_{1}, \\
& F_{A} l_{2}+F_{B} l_{2}=F_{D} l_{2}+F_{E} l_{2} .
\end{aligned}
$$

The last equations (5) can be written as

$$
\begin{aligned}
& F_{A}+F_{E}=F_{B}+F_{D}, \\
& F_{A}+F_{B}=F_{D}+F_{E},
\end{aligned}
$$

wherefrom, by subtracting, we get

$$
\begin{aligned}
F_{B} & =F_{E} \\
F_{A} & =F_{D} .
\end{aligned}
$$

The relations (7) lead to

$$
\begin{aligned}
& \left(l_{1} \psi-l_{2} \theta\right)\left(k_{1}+2 k_{2} z\right)=0, \\
& \left(l_{1} \psi+l_{2} \theta\right)\left(k_{1}+2 k_{2} z\right)=0 .
\end{aligned}
$$

\subsubsection{The case $k_{1}+2 k_{2} z=0$}

In this situation, one obtains $z=-k_{1} /\left(2 k_{2}\right)$. Replacing in the equation (3), one gets

$$
4 k_{2}\left(l_{1}^{2} \psi^{2}+l_{2}^{2} \theta^{2}\right)=\frac{m g k_{2}+k_{1}^{2}}{k_{2}} .
$$

Since $l_{1}^{2} \psi^{2}+l_{2}^{2} \theta^{2} \geq 0$ it results that $m g k_{2}+k_{1}^{2} \geq 0$, wherefrom $k_{2} \geq-k_{1}^{2} /(m g)$. But $l_{1}^{2} \psi^{2}+l_{2}^{2} \theta^{2}$ is a small value (from the hypothesis that the angles $\psi$ and $\theta$ are small) and from here we deduce that $k_{2} \simeq-k_{1}^{2} /(\mathrm{mg})$. It means that there exists a relation between the parameters $k_{1}, k_{2}$ and $m$. Moreover, the expression (9) defines an ellipse in the $\psi \theta$ plan. In this case, there is a domain around the point $(z, \psi, \theta)=\left(-k_{1} /\left(2 k_{2}\right), 0,0\right)$ in which the system is at equilibrium. We can say that in this situation there exist an infinity of equilibrium positions which defines an open neighborhood around the point $\left(-k_{1} /\left(2 k_{2}\right), 0,0\right)$. These equilibrium positions are positions of indifferent equilibrium.

\subsubsection{The case $k_{1}+2 k_{2} z \neq 0$}

This case is characterized by $l_{1} \psi-l_{2} \theta=0$ and $l_{1} \psi+l_{2} \theta=$ 0 , wherefrom $\psi=0$ and $\theta=0$. From the equation (3) we obtain

$$
4 k_{2} z^{2}+4 k_{1} z-m g=0,
$$

the discriminant of which is $\Delta=\sqrt{16 k_{1}^{2}+16 k_{2} m g}$. There exist equilibrium positions if and only if $\Delta \geq 0$, which implies $k_{2} \geq-k_{1}^{2} /(\mathrm{mg})$. In addition, the equilibrium positions are described by

$$
\begin{aligned}
& z_{1}=\frac{-k_{1}+\sqrt{k_{1}^{2}+k_{2} m g}}{2 k_{2}}, \\
& z_{2}=\frac{-k_{1}-\sqrt{k_{1}^{2}+k_{2} m g}}{2 k_{2}} .
\end{aligned}
$$

If $k_{2}>0$, then $z_{1}>0$ and $z_{2}<0$, and if $k_{2}<0$, then $z_{1}>0$ and $z_{2}>0$.

\subsection{All the elastic forces are vertical descendent}

The relations (5) and (9) become now

$$
\begin{aligned}
& F_{A}+F_{B}+F_{D}+F_{E}+m g=0, \\
& F_{A} l_{1}+F_{E} l_{1}=F_{B} l_{1}+F_{D} l_{1}, \\
& F_{A} l_{2}+F_{B} l_{2}=F_{D} l_{2}+F_{E} l_{2}
\end{aligned}
$$


and

$$
4 k_{2}\left(l_{1}^{2} \psi^{2}+l_{2}^{2} \theta^{2}\right)=\frac{-m g k_{2}+k_{1}^{2}}{k_{2}}
$$

respectively.

Since the left-hand side term of the equation (13) is positive, it results the expression

$$
\frac{1}{k_{2}} \geq \frac{m g}{k_{1}^{2}}>0
$$

The rest of discussion is similar to that from the paragraph 4.1.1.

\subsection{Three elastic forces have the same sense and one opposite sense}

For the clarity we will assume that the forces $F_{A}, F_{B}$ and $F_{D}$ are vertical ascendent, while the force $F_{E}$ is vertical descendent. The rest of cases are similar to this one. The second and the third equations (5) read now

$$
\begin{aligned}
& -F_{A}+F_{B}+F_{D}+F_{E}=0, \\
& -F_{A}-F_{B}+F_{D}-F_{E}=0,
\end{aligned}
$$

wherefrom

$$
\begin{aligned}
& F_{A}=F_{D}, \\
& F_{B}=-F_{E} .
\end{aligned}
$$

The first relation (16) leads to

$$
\left(l_{1} \psi+l_{2} \theta\right)\left(k_{1}+2 k_{2} z\right)=0 \text {, }
$$

while the second one offers

$$
k_{2} z^{2}+k_{1} z+\left(l_{1} \psi-l_{2} \theta\right)^{2}=0 .
$$

The first equation (5) becomes now

$$
F_{A}+F_{B}+F_{D}-F_{E}=m g,
$$

so that we get

$$
\left(k_{1}+k_{2} z\right)\left(z+l_{1} \psi-l_{2} \theta\right)+k_{2}\left(l_{1} \psi+l_{2} \theta\right)^{2}=m g .
$$

4.3.1. The case $k_{1}+2 k_{2}=0$

Equation (18) reads now

$$
\left(l_{1} \psi-l_{2} \theta+\sqrt{\frac{k_{1}^{2}}{4 k_{2}}}\right)\left(l_{1} \psi-l_{2} \theta-\sqrt{\frac{k_{1}^{2}}{4 k_{2}}}\right),
$$

which defines two parallel lines in the $\psi-\theta$ plane. It also results

$$
k_{1}+k_{2} z=\frac{k_{1}}{2} ;
$$

hence, the expression (20) may be put in the form

$$
\frac{k_{1}}{2}\left(z+l_{1} \psi-l_{2} \theta\right)+k_{2}\left(l_{1} \psi+l_{2} \theta\right)^{2}=m g
$$

and since $\left(l_{1} \psi+l_{2} \theta\right)^{2} \simeq 0$, we deduce the relation

$$
\iota_{1} \psi-l_{2} \theta=\frac{2 m g k_{2}+k_{1}^{2}}{2 k_{1} k_{2}}
$$

that is, a straight line in the $\psi-\theta$ plane.

\subsubsection{The case $l_{1} \psi+l_{2} \theta=0$}

In this situation, one obtains the relation

$$
k_{2} z^{2}+k_{1} z+4 l_{1}^{2} \psi^{2}=0
$$

which may be also written as

$$
\left(z+\frac{k_{1}}{2 k_{2}}\right)^{2}+\frac{4 l_{1}^{2}}{k_{2}} \psi^{2}=\frac{k_{1}^{2}}{4 k_{2}^{2}} .
$$

The last equation defines in the $\psi-\theta$ plane an ellipse if $k_{2}>0$ or a hyperbola if $k_{2}<0$.

Moreover, the expression (20) leads now to

$$
\left(k_{1}+k_{2} z\right)\left(z+l_{1} \psi-l_{2} \theta\right)=m g .
$$

Since $\theta=-l_{1} \psi / t_{2}$, that is

$$
l_{1} \psi-l_{2} \theta=2 l_{1} \psi
$$

and making the approximation

$$
z+l_{1} \psi-l_{2} \theta \simeq z
$$

one obtains the equation

$$
k_{2} z^{2}+k_{1} z=m g
$$

with 0,1 or 2 real solutions depending on the sign of

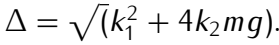


4.4. Two elastic forces are vertical ascendent, while the other two are vertical descendent

According to the theorem of moment of momentum, the only possibility for the equilibrium is that the forces situated on the same diagonal have the same sense. For the clarity, we will assume that the forces $F_{A}$ and $F_{D}$ are vertical ascendent, while the forces $F_{B}$ and $F_{E}$ are vertical descendent. The second and the third relations (5) leads now to

$$
\begin{aligned}
& F_{A}+F_{B}=F_{D}+F_{E}, \\
& F_{A}+F_{E}=F_{B}+F_{D},
\end{aligned}
$$

wherefrom

$$
\begin{aligned}
F_{B} & =F_{E}, \\
F_{A} & =F_{D} .
\end{aligned}
$$

The first equation (31) leads to

$$
\left(l_{1} \psi-l_{2} \theta\right)\left(k_{1}+2 k_{2} z\right)=0,
$$

while the second one gets

$$
\left(l_{1} \psi+l_{2} \theta\right)\left(k_{1}+2 k_{2} z\right)=0 .
$$

From the first equation (5) results

$$
F_{A}+F_{D}=F_{B}+F_{E}+m g,
$$

wherefrom one obtains

$$
8 k_{2} l_{1} l_{2} \psi \theta=m g,
$$

that is a hyperbola in the $\psi-\theta$ plane.

\section{Stability of the equilibrium positions}

\subsection{All the elastic forces are vertical ascendent}

Denoting $z=\xi_{1}, \psi=\xi_{2}, \theta=\xi_{3}, \dot{z}=\xi_{4}, \dot{\psi}=\xi_{5}, \dot{\theta}=\xi_{6}$, the equations of motion may be put in the form of a six first order nonlinear differential equations

$$
\begin{aligned}
& \dot{\xi}_{1}=\xi_{4}, \dot{\xi}_{2}=\xi_{5}, \dot{\xi}_{3}=\xi_{6}, \\
& \dot{\xi}_{4}=-\frac{4 k_{1} \xi_{1}}{m}-\frac{4 k_{2}\left(\xi_{1}^{2}+l_{1}^{2} \xi_{2}^{2}+l_{2}^{2} \xi_{3}^{2}\right)}{m}-g, \\
& \dot{\xi}_{5}=-\frac{4 k_{1} l_{1}^{2} \xi_{2}}{J_{x}}-\frac{8 k_{2} \xi_{1} l_{1}^{2} \xi_{2}}{J_{x}}, \\
& \dot{\xi}_{6}=-\frac{4 k_{1} l_{2}^{2} \xi_{3}}{J_{y}}-\frac{8 k_{2} \xi_{1} l_{2}^{2} \xi_{3}}{J_{y}} .
\end{aligned}
$$

Further on, we denote by $j_{k l}$ the partial derivatives $j_{k l}=\partial f_{j} / \partial \xi_{l}$, where $f_{j}$ marks the right-hand terms of the expressions (37), and $k, l=\overline{1,6}$. One calculates

$$
\begin{gathered}
j_{14}=1, \\
j_{25}=1, \\
j_{36}=1, \\
j_{41}=-\frac{4 k_{1}}{m}-\frac{8 k_{2} \xi_{1}}{m}, \\
j_{42}=-\frac{8 k_{2} l_{1}^{2} \xi_{2}}{m}, \\
j_{43}=-\frac{8 k_{2} l_{2}^{2} \xi_{3}}{m}, \\
j_{51}=-\frac{8 k_{2} l_{1}^{2} \xi_{2}}{J_{x}}, \\
j_{52}=-\frac{4 k_{1} l_{1}^{2}}{J_{x}}-\frac{8 k_{2} l_{1}^{2} \xi_{1}}{J_{x}}, \\
j_{61}=-\frac{8 k_{2} l_{2}^{2} \xi_{3}}{J_{y}}, \\
j_{63}=-\frac{4 k_{1} l_{2}^{2}}{J_{y}}-\frac{8 k_{2} l_{2}^{2} \xi_{1}}{J_{y}},
\end{gathered}
$$

the rest of $j_{k l}$ being zero.

The characteristic equation reads ( $\lambda$ being the complex rate of growth)

$$
\left|\begin{array}{cccccc}
-\lambda & 0 & 0 & 1 & 0 & 0 \\
0 & -\lambda & 0 & 0 & 1 & 0 \\
0 & 0 & -\lambda & 0 & 0 & 1 \\
j_{41} & j_{42} & j_{43} & -\lambda & 0 & 0 \\
j_{51} & j_{52} & 0 & 0 & -\lambda & 0 \\
j_{61} & 0 & j_{63} & 0 & 0 & -\lambda
\end{array}\right|=0
$$

wherefrom

$$
\lambda^{6}+A \lambda^{4}+B \lambda^{2}+C=0
$$

with

$$
\begin{aligned}
& A=-\left(j_{41}+j_{52}+j_{63}\right), \\
& B=\left(j_{52} j_{63}+j_{41} j_{63}+j_{41}+j 41 j_{52}-j_{43} j_{61}-j_{42} j_{51}\right), \\
& C=-j_{41} j_{52} j_{63}+j_{43} j_{52} j_{61}+j_{42} j_{51} j_{63} .
\end{aligned}
$$

Putting $u=\lambda^{2}$, one obtains the third degree equation

$$
u^{3}+A u^{2}+B u+C=0 .
$$

According to the Descartes theorem [27, 28], this equations has no positive solution if and only $A>0$ 
$B>0$ and $C>0$. With the change of variable $u=$ $v-A / 3$, the equation becomes

$$
v^{3}+a v+b=0
$$

with

$$
\begin{aligned}
& a=B-\frac{A^{2}}{3} \\
& b=\frac{2 A^{3}}{27}-\frac{A B}{3}+C .
\end{aligned}
$$

The equation (46) has three real roots if and only if [28] its discriminant $\Delta=4 a^{3}+27 b^{2}$ is negative, that is

$$
\Delta=4 B^{3}-A^{2} B^{2}+27 C^{2}+4 A^{3} C-18 A B C \leq 0 .
$$

If we consider the equilibrium positions described by $\psi=$ $\xi_{2}=0, \theta=\xi_{3}=0$, while $z$ is given by the relations (11), then the characteristic equation takes the simpler form

$$
\left(j_{41}-\lambda^{2}\right)\left(j_{52}-\lambda^{2}\right)\left(j_{63}-\lambda^{2}\right)=0
$$

and the solutions are $\lambda= \pm \sqrt{j_{41}}, \lambda= \pm \sqrt{j_{52}}$, and $\lambda=$ $\pm \sqrt{j_{63}}$.

The equilibrium position is stable (in fact, simply stable) if and only if $j_{41}<0, j_{52}<0$, and $j_{63}<0$. These conditions are equivalent with the condition $k_{1}+2 k_{2} z_{e q}>$ 0 , where $z_{e q}$ stays for the value of the coordinate $z$ in the equilibrium position, value given by one of the relations (11). Replacing the values given by (11) in the stability condition, one gets $\pm \sqrt{k_{1}^{2}+k_{2} m g}>0$, which holds true only for the value $z_{1}$. In conclusion, the only stable position is given by $z_{1}$.

The small oscillations around the equilibrium position are harmonic motions given by [27]

$$
\begin{aligned}
& z=z_{0} \cos \left(\sqrt{\left|j_{41}\right|} t+\varphi_{1}\right), \\
& \psi=\psi_{0} \cos \left(\sqrt{\left|j_{52}\right|} t+\varphi_{2}\right), \\
& \theta=\theta_{0} \cos \left(\sqrt{\left|j_{52}\right|} t+\varphi_{3}\right),
\end{aligned}
$$

where $z_{0}, \psi_{0}, \theta_{0}, \varphi_{1}, \varphi_{2}$. and $\varphi_{3}$ are constants of integration with the values determined from the initial conditions.

The situation defined by all vertical descendent force is completely similar; we only have to change the sign in the previous expressions.

\subsection{Three elastic forces are vertical ascendent, while one is vertical descendent}

Considering again that the forces $F_{A}, F_{B}$ and $F_{D}$ are vertical ascendent, and the force $F_{E}$ is a vertical descendent one, one obtains the equations of motion

$$
\begin{aligned}
& m \ddot{z}=F_{A}+F_{B}+F_{D}-F_{E}-m g, \\
& J_{x} \ddot{\psi}=-F_{A} l_{1}+F_{B} l_{1}+F_{D} l_{1}+F_{E} l_{1}, \\
& J_{y} \ddot{\theta}=-F_{A} l_{2}-F_{B} l_{2}+F_{D} l_{2}-F_{E} l_{2},
\end{aligned}
$$

wherefrom, using the same notations as in paragraph 5.1,

$$
\begin{aligned}
\dot{\xi}_{1} & =\xi_{4}, \dot{\xi}_{2}=\xi_{5}, \dot{\xi}_{3}=\xi_{6}, \\
\dot{\xi}_{4} & =\frac{2 k_{1}\left(\xi_{1}+l_{1} \xi_{2}-l_{2} \xi_{3}\right)}{m}+\frac{2 k_{2} \xi_{1}^{2}}{m}+\frac{4 k_{2} \xi_{1}\left(l_{1} \xi_{2}-l_{2} \xi_{3}\right)}{m} \\
& +\frac{2 k_{2}\left(l_{1} \xi_{2}+l_{2} \xi_{3}\right)}{m}-g, \\
\dot{\xi}_{5} & =\frac{2 k_{1}\left(\xi_{1}+l_{1} \xi_{2}-l_{2} \xi_{3}\right)}{J_{x}}+\frac{2 k_{2} \xi_{1}^{2}}{J_{x}} \\
& +\frac{4 k_{2} \xi_{1}\left(l_{1} \xi_{2}-l_{2} \xi_{3}\right)}{J_{x}}+\frac{2 k_{2}\left(l_{1} \xi_{2}+l_{2} \xi_{3}\right)^{2}}{J_{x}}, \\
\dot{\xi}_{6} & =-\frac{2 k_{1}\left(\xi_{1}-l_{1} \xi_{2}-l_{2} \xi_{3}\right)}{J_{y}}-\frac{2 k_{3} \xi_{1}^{2}}{J_{y}}+\frac{4 k_{2} \xi_{1}\left(l_{1} \xi_{2}+l_{2} \xi_{3}\right)}{J_{y}} \\
& -\frac{2 k_{2}\left(l_{1} \xi_{2}-l_{2} \xi_{3}\right)^{2}}{J_{y}} .
\end{aligned}
$$

The discussion is completely similar to that at the paragraph 5.1.

\section{The influence of damping}

In this case, the system (53) becomes

$$
\begin{aligned}
& \dot{\xi}_{1}=\xi_{4}, \dot{\xi}_{2}=\xi_{5}, \dot{\xi}_{3}=\xi_{6}, \\
& \dot{\xi}_{4}=-\frac{4 k_{1} \xi_{1}}{m}-\frac{4 k_{2}\left(\xi_{1}^{2}+l_{1}^{2} \xi_{2}^{2}+l_{2}^{2} \xi_{3}^{2}\right)}{m}-\frac{4 c \xi_{4}}{m}-g, \\
& \dot{\xi}_{5}=-\frac{4 k_{1} l_{1}^{2} \xi_{2}}{J_{x}}-\frac{8 k_{2} \xi_{1} l_{1}^{2} \xi_{2}}{J_{x}}, \\
& \dot{\xi}_{6}=-\frac{4 k_{1} l_{2}^{2} \xi_{3}}{J_{y}}-\frac{8 k_{2} \xi_{1} l_{2}^{2} \xi_{3}}{J_{y}},
\end{aligned}
$$

where $c$ is the damping coefficient.

The only parameter that changes its value is

$$
j_{44}=-\frac{4 c}{m}<0
$$

The characteristic equation becomes now 


$$
\left|\begin{array}{cccccc}
-\lambda & 0 & 0 & 1 & 0 & 0 \\
0 & -\lambda & 0 & 0 & 1 & 0 \\
0 & 0 & -\lambda & 0 & 0 & 1 \\
j_{41} & j_{42} & j_{43} & j_{44}-\lambda & 0 & 0 \\
j_{51} & j_{52} & 0 & 0 & -\lambda & 0 \\
j_{61} & 0 & j_{63} & 0 & 0 & -\lambda
\end{array}\right|=0,
$$

wherefrom

$$
\left|\begin{array}{ccc}
j_{41}+j_{44} \lambda-\lambda^{2} & j_{42} & j_{43} \\
j_{51} & j_{52}-\lambda^{2} & 0 \\
j_{61} & 0 & j_{63}-\lambda^{2}
\end{array}\right|=0,
$$

so that one gets

$$
\lambda^{6}+A \lambda^{5}+B \lambda^{4}+C \lambda^{3}+D \lambda^{2}+E \lambda+F=0 .
$$

The roots of the last equation (and therefore, the stability of the equilibrium positions) may be discussed with the aid of the Routh-Hurwitz criterion.

In the real cases, the damper is not presented and the damping appears from the natural characteristic of the spring. Hence, the values of the coefficient $c$ are very small and the terms with odd powers in equation (57) can be neglected.

\section{Numerical example}

As a numerical example we consider the following realistic values: $m=200 \mathrm{~kg}, g=10 \mathrm{~m} / \mathrm{s}^{2}, l_{1}=0.45 \mathrm{~m}, l_{2}=$ $0.3 \mathrm{~m}, k_{1}=10^{5} \mathrm{~N} / \mathrm{m}, k_{2}=10^{7} \mathrm{~N} / \mathrm{m}^{2}, J_{x}=13.5 \mathrm{kgm}^{2}$, $J_{y}=6 \mathrm{kgm}^{2}$

One obtains the equation

$$
20000 z^{2}+200 z-1=0,
$$

with the solutions $z_{1}=0.00366 \mathrm{~m}$ and $z_{2}=-0.01366 \mathrm{~m}$. The partial derivatives are $j_{41}=-3464, j_{52}=-10392$, and $j_{63}=-10392$ for $z_{1}$, respective $j_{41}=3464, j_{52}=$ 10392, and $j_{63}=10392$ for $z_{2}$. The eigenpulsations for the small oscillations arround the simply stable position of equilibrium defined by $z_{1}$ are $\omega_{1}=58.86 \mathrm{~Hz}$ for $z$, $\omega_{2}=101.94 \mathrm{~Hz}$ for $\psi$, and $\omega_{3}=101.94 \mathrm{~Hz}$ for $\theta$.

\section{Conclusions}

Our paper presented a model for the suspension of an engine using four identic springs of quadratic characteristic. We proved that there exist situations, described by particular values of the parameters $k_{1}, k_{2}$ and $m$ (linking therefore the elastic and the inertial properties of the system), situation for which the systems presents an infinite number of equilibrium positions. This result is a paradox having as causes the linear approximations for the rotational angles about the $C x$ and $C y$-axes, and the quadratic characteristic of the springs. The second paradox is defined by the equilibrium position, in fact by their stability. The present study showed that the simply stable position of equilibrium is that for which the springs are stretched, not the position in which the springs are compressed. The reader can easily proved that for both positions of equilibrium the elastic forces are positive, i.e. the forces in the springs are vertical ascendent (as they have to be to equilibrate the weight of the engine). The small oscillations around the equilibrium position are harmonic ones and we determined the eigenpulsations of these oscillations. The method presented here, based on dynamical systems for the study of the equilibrium, is equivalent with the method of the normal forms, not detailed in this paper. The equivalence is assured by the fact that the eigenvalues of a matrix $A$ are the same but with signs changed as those of the matrix $-\mathrm{A}$.

\section{References}

[1] Pandrea N., Pârlac S., Popa D., Models for the study of the vibrations of automobiles, Tiparg, Piteşti, 2001 (in Romanian).

[2] Tongue B. H., Principles of Vibrations, Oxford University Press, Oxford, 2001

[3] Schmitz T. L., Smith K. S., Mechanical Vibrations: Modeling and Measurement, Springer, New York, 2011

[4] Wirsching P. H., Paez T. L., Ortiz K., Random Vibrations: Theory and Practice, Dover Books on Physics, Dover, 2006

[5] Meirovitch L., Fundamental of Vibrations, Waveland Press, Inc, Long Grove, 2010

[6] Geng Z., Chen J., Barry Hull J., Analysis of engine vibration and design of an applicable diagnosing approach, International Journal of Mechanical Sciences, Volume 45, Issue 8, 2003, 1391-1410

[7] Junhong Z., Jun $H$., CAE process to simulate and optimise engine noise and vibration, Mechanical Systems and Signal Processing, Volume 20, Issue 6, 2006, 1400-1409

[8] Carlucci A. P., Chiara F. F., Laforgia D., Analysis of the relation between injection parameter variation and block vibration of an internal combustion diesel 
engine, Journal of Sound and Vibration, Volume 295, Issues 1-2, 2006, 141-164

[9] Stănescu N.-D., Popa D., The Vibrations of the Engine with neo-Hookean Suspension, Acoustics and Vibration of Mechanical Structures, AVMS 2013, Timişoara, 2013

[10] Kilic, M., Yazicioglu, Y., Kurtulus, D., F., Synthesis of a torsional spring mechanism with mechanically adjustable stiffness using wrapping cams, Mechanism and Machine Theory 57, 2012, 27-39

[11] Luo, J., Luo, Z., Chen, S., Tong, L., Wang, M., Y., A new level set method for systematic design of hinge-free compliant mechanisms, Comput. Methods Appl. Mech. Engrg. 198, 2008, 318-331

[12] Champion, R., Champion, W., L., The extension and oscillation of a non-Hooke's law spring, European Journal of Mechanics - A/Solids, 26, 2, (2007), 286297

[13] Vehar Jutte, C., Generalized Synthesis Methodology of Nonlinear Springs for Prescribed LoadDisplacement functions, Doctoral Thesis, University of Michigan, 2008

[14] Vehar Jutte, C., Kota, S., Design of Nonlinear Springs for Prescribed Load-Displacement Functions, Journal of Mechanical Design 130, 2008, 081403, 1-10

[15] Worden, K, Hickey, D., Harron, M., Adams, D., E., Nonlinear system identification of automotive dampers: A timeandfrequency-domainanalysis, Mechanical Systems and Signal Processing, 23, (2009), 104-126

[16] Poussot-Vassal, C., Spelta, C., Sename, O., Savaresi, S., M., Dugard, L., Survey and performance evaluation on some automotive semi-active suspension control methods: A comparative study on a single-corner model, Annual Reviews in Control 36, 2012, 148-160

[17] Yang, J., Zolotas, A., Chenc, W.-H., Michail, K., Li, S., Robust control of nonlinear MAGLEV suspension system with mismatched uncertainties via DOBC approach, ISA Transactions 50, 2011, 389-396
[18] Lee, D.-C., Han, C.-S., CAE (computer aided engineering)driven durability model verification for the automotive structure development, Finite Elements in Analysis and Design 45, 2009, 324-332

[19] Huang, C.-J., Lin, J.-S., Chen, C.-C., Road-adaptive algorithm design of half-car active suspension system, Expert Systems with Applications 37, 2010, 43924402

[20] Huang, S.-J., Chen, H.-Y., Adaptive sliding controller with self-tuning fuzzy compensation for vehicle suspension control, Mechatronics 16, 2006, 607-622

[21] Koch, G., Fritsch, O., Lohmann, B., Potential of low band width active suspension control with continuously variable damper, Control Engineering Practice 18, 2010, 1251-1262

[22] Ibrahim, R., A., Recent advances in nonlinear passive vibration isolators, Journal of Sound and Vibration 314, 2008, 371-452

[23] Wei, W., Yuling, S., Nonlinear vibration semiactive control of automotive steering using magnetorheological damper, Meccanica 47, 2012, 2027-2039

[24] Surace, C., Worden, K,m Tomlinson, G., R., An Improved Nonlinear Model for an Automotive Shock Absorber, Nonlinear Dynamics 3, 1992, 413-429

[25] Ando, Y., Suzuki, M., Control of active suspension system using the singular perturbation method, Control Engineering Practice 331, 2012, 5824-5837

[26] Lin, J.-S., Kanellakopoulos, I., Nonlinear Design of Active Suspensions, IEEE Control Systems Magazine 17, 1995, 45-49

[27] Stănescu N.-D., Munteanu L., Chiroiu V., Pandrea N., Dynamical Systems. Theory and Applications, The Publishing House of the Romanian Academy, Bucharest 2007, 2011, 2013 (in Romanian)

[28] Teodorescu P., Stănescu N.-D., Pandrea N., Numerical Analysis with Applications in Mechanics and Engineering, Wiley, Hoboken, 2013 